\title{
Diallel crosses reveal patterns of variation in fruit-set, seed mass, and seed number in Asclepias incarnata
}

\author{
SARA R. LIPOW* $† \&$ ROBERT WYATT† \\ $\dagger$ Department of Botany, University of Georgia, Athens, GA 30602, U.S.A. and $\ddagger$ Institute of Ecology, \\ University of Georgia, Athens, GA 30602, U.S.A.
}

\begin{abstract}
Plants from each of two populations of Asclepias incarnata ssp. incarnata were hand-pollinated in diallel crosses and seed number per fruit, mean individual seed mass per fruit and fruit-set were determined for the parental plants. Two diallels were performed on the glasshouse-grown plants, one involving six plants from one population and the other involving five plants from the second population. The contributions to total phenotypic variation from joint, maternal, paternal, and two types of interaction sources of variation were then estimated. Variance attributable to maternal effects, reflecting differences between maternal plants resulting from environmental or genetic factors or both, comprised $31.3-68.4 \%$ of total variance in seed mass, $20.5-37.1 \%$ of variance in seed number, and $0.7-7.5 \%$ of variance in fruit-set. Interaction effects that depended on the direction of the cross accounted for $7.3-46.5 \%$ of variance in seed mass and $31.4-39.1 \%$ of variance in seed number. These interaction effects are consistent with the hypothesis that maternal plants provision resources differently to seeds depending on the specific genotypes of the embryos they contain. In the analysis of fruit-set, interaction effects independent of the direction of the cross (combined variance) explained $22.9-78.0 \%$ of phenotypic variance. These effects could have several causes, including inbreeding depression, shared incompatibility alleles, or 'control' of fruit-set by zygotes via hormonal signals or patterns of resource acquisition.
\end{abstract}

Keywords: bio model, female choice, functional gender, seed abortion, selection response, sexual selection.

\section{Introduction}

The reproductive success of a plant is determined by the number of fruits it produces and the quantity and quality of seeds in those fruits. Significant phenotypic variation in fruit-set (Bookman, 1984; Marshall \& Ellstrand, 1986; Mazer, 1987a; Morse \& Schmitt, 1991) and seed number and seed mass (Antonovics \& Schmitt, 1986; Marshall \& Ellstrand, 1986; Mazer, 1987a; Schwaegerle \& Levin, 1990; Waser et al., 1995) is extremely common in natural populations. Because these traits are directly linked to fitness, selection will act very quickly on any portion of the phenotypic variation

*Correspondence and present address: Department of Forest Sciences, Oregon State University, Corvallis, OR 97331, U.S.A.

E-mail: slipow/r6pnw-corvallis@fs.fed.us

$\S$ Present address: Highlands Biological Station, P.O. Box 580, Highlands, NC 28741, U.S.A.

E-mail: wyatt@dogwood.botany.uga.edu that is unconstrained by linkage, pleiotropy, or strong genotype by environment interactions (Falconer, 1989).

In hermaphroditic plants, reproductive success results from both fruits sired (male function) and fruits matured (female function). These functions can be under different selective pressures, and the variance may differ between them (Lyons et al., 1989). For example, variation in a characteristic such as inflorescence size may differentially influence pollen donation and receipt and, thus, have gender-specific effects (Morgan, 1994). To understand the causes of variation in this characteristic both male and female functions must be evaluated.

Variance in male and female function can also differ for traits, such as fruit- and seed-set, that are affected by postpollination processes (Wilson et al., 1994). The evolution of these traits, however, is determined not only by the variance in the gender functions of the parental plants, but also by variation in their diploid progeny, as well as by interactions between the progeny 
and parental genotypes. Thus, estimates of several components of phenotypic variation are required to evaluate the potential response of these traits to selection (Morgan, 1994). In this regard, studies employing diallel crossing designs are among the most informative, because these designs allow phenotypic variation to be partitioned into as many as six components. These components represent the contribution to phenotypic variation by genes expressed jointly in the male or female parent, from maternal parents, from paternal parents, and from two types of parental interactions (Cockerham \& Weir, 1977). They are described in detail below. Six is the maximum number of components that can be estimated from a crossing design involving only a progeny generation and an unmeasured parental generation (Cockerham \& Weir, 1977; Waser et al., 1995).

Here we describe a study in which diallel crosses were performed to analyse variation in three postpollination traits in plants from two populations of Asclepias incarnata ssp. incarnata: (i) the proportion of pollinated flowers that matured into fruits; (ii) seed number per fruit; and (iii) mean individual seed mass per fruit. The six components of phenotypic variation were estimated for these traits. Additionally, the correlation between male and female function was evaluated, and levels of variation between individuals with respect to male and female function were compared.

Several previous studies have partitioned phenotypic variation for seed mass (Antonovics \& Schmitt, 1986; Schwaegerle \& Levin, 1990; Biere, 1991; Montalvo \& Shaw, 1994; Waser et al., 1995; Lyons, 1996), seed number (Biere, 1991; Waser et al., 1995; Lyons, 1996) and fruit-set (Morse \& Schmitt, 1991; Lyons, 1996) into the same six variance components, with the goal of understanding the evolutionary response of these traits to selection. In these studies, most of the observed variation was attributed to maternal and environmental effects. The milkweeds we examined, however, have some unique features that we predicted would increase our ability to detect interesting paternal or interaction effects for seed number and fruit-set. In milkweeds, fruit-set is uniformly low, rarely exceeding $7 \%$ in natural populations (Wilbur, 1976) and typically averaging $<50 \%$ after cross-pollinations under ideal glasshouse conditions (Wyatt \& Broyles, 1994; Ivey et al., 1999). Because many pollinated flowers do not mature fruit, there appears to be ample opportunity for genetic interactions to govern fruit-set. Moreover, milkweed pollen is enclosed in discrete sacs termed 'pollinia', and each pollinium contains enough pollen grains to fertilize all of the ovules in an ovary. The pollen tubes from one pollinium travel as a cord through the transmitting tract (Wyatt \& Broyles, 1994). We suspected that this unusual pollination system would create a more uniform environment for the pollen during the pregamic phase and, consequently, decrease environmental variation for seed number.

\section{Methods}

\section{Species description}

Asclepias incarnata L. ssp. incarnata is a perennial herb that grows in wetlands from Florida north to Manitoba and west to Utah and New Mexico (Ivey et al., 1999). Individual plants consist of one or more stems that emerge from a root crown. Each stem produces many paired umbels, which contain numerous pink to rose flowers that are pollinated by wasps and bees (Wyatt \& Broyles, 1994). Most flowers on an umbel open simultaneously, but different umbels on a plant may open over a period of four weeks or longer. The fruit is a follicle that typically contains 45-65 wind-dispersed seeds. Natural fruit-set in A. incarnata averages about 7\% (Wilbur, 1976); this is low compared to most flowering plants, but high relative to other milkweeds (Wyatt \& Broyles, 1994). Our study plants came from northern Virginia, where populations contain a mixture of self-fertile and selfsterile individuals (Lipow, 1998). Nevertheless, in natural populations the plants are largely outcrossed, as indicated by multilocus estimates of outcrossing rates that range from $88 \%$ to $99 \%$ (Ivey et al., 1999).

\section{Diallel crosses}

Seeds of $A$. incarnata ssp. incarnata were collected from two natural populations in northern Virginia (Frederick and Shenandoah Cos). Six seeds from the Frederick population and five seeds from the Shenandoah population, each taken from a different maternal plant, were sown and the seedlings grown to maturity in a pollinator-free glasshouse (Botany Department, University of Georgia). Two diallel crosses were then performed, one involving plants from the Frederick population (D1) and one involving plants from the Shenandoah population (D2). Self-pollinations were excluded, because many individuals of $A$. incarnata from these populations are known to be self-sterile (Lipow, 1998). Conducting the diallels in the glasshouse reduced environmental variation and, thereby, increased the likelihood of detecting genetic sources of variation.

Because many glasshouse-grown plants of $A$. incarnata will not flower in the first year following germination, we used three-year-old plants. To minimize differences in resource status between these plants, they were replanted in April 1997, following removal of all above-ground biomass. Each root crown was potted in an 11.4-L pot 
containing a mixture of pine-bark, vermiculite, perlite, lime and micronutrients. The pots were randomly positioned on glasshouse benches, and their positions were changed weekly during the flowering and fruiting periods. Pollinations for D1 were carried out from 20 to 29 July 1997 and for D2, 23 June to 30 July 1997. The order of pollination for different crosses was randomized. All plants were watered twice daily by an automatic drip-line irrigation system and fertilized semiweekly, once with Peters $^{\mathrm{TM}}$ 20:10:20 and once with Peters ${ }^{\mathrm{TM}}$ 10:30:20 plus added iron. A 14-h photoperiod was maintained using supplemental lighting.

The resulting plants were large and vigorous, and each produced at least four flowering shoots. Each shoot consisted of a main stem with several secondary stems. These secondary stems usually branched several more times and produced many inflorescences. We pollinated only a single umbel per secondary branch, however, to minimize the potential for resource competition between umbels.

For each of the 30 and 20 cells in D1 and D2, respectively, we hand-pollinated a total of 25-40 flowers on five to eight umbels. Five randomly selected flowers per umbel were pollinated, with each flower on the umbel receiving a single pollinium from the same, randomly assigned pollen donor. Fruits were harvested at maturity and fruit-set was calculated. Additionally, seeds from four randomly selected fruits per cell of the diallel were examined, although in a few cases $(<10 \%)$ fewer than four fruits matured. Whenever possible, the fruits selected were from different umbels. The average mass of individual seeds (found by measuring 10 randomly selected seeds per fruit) and seed number (estimated by the total seed mass divided by the individual seed mass) were determined after removal of the comas from the seeds.

\section{Statistical analysis}

To examine whether relationships existed among the three postpollination traits, partial correlation coefficients were calculated from multivariate analyses (MANOVA statement of GLM; SAS Institute, 1987) in which pairs of traits were treated as dependent variables. For seed number and mass, the models included the effects of female plant, male plant, and the female-male interaction. For fruit-set with seed number and seed mass, the models included only the effect of the female plant.

The data for each postpollination trait were then analysed separately using the 'bio' model of Cockerham \& Weir (1977). This model partitions total phenotypic variance $\left(V_{\text {phenotypic }}\right)$ into six variance components. We followed the parameterization of Montalvo \& Shaw (1994), who represented $V_{\text {phenotypic }}$ in terms of 'causal' components (see Falconer, 1989), but adopted the terminology introduced by Lyons (1996):

$$
\begin{aligned}
V_{\text {phenotypic }}= & V_{\text {maternal }}+V_{\text {paternal }}+V_{\text {joint }}+V_{\text {combined }} \\
& +V_{\text {interaction }}+V_{\text {environmental }} .
\end{aligned}
$$

$V_{\text {maternal }}$ represents maternal variation caused by environmental, cytoplasmic and nuclear effects specific to maternal function, other than those from nuclear genes. $V_{\text {paternal }}$ is analogous to $V_{\text {maternal }}$. $V_{\text {joint }}$ estimates the joint contribution of parents through male or female function. $V_{\text {combined }}$ represents effects specific to particular parental combinations, regardless of the direction of the cross (i.e. symmetrical effects). It may result from interactions of alleles at individual nuclear loci. $V_{\text {interaction }}$ represents the asymmetrical interaction of offspring and parental genomes and includes variation in performance of particular parental combinations when sex roles of the parents are reversed. Finally, $V_{\text {environmental }}$ represents the variance of residuals within observations for the cells of the diallel.

Parameters were estimated by restriction maximum likelihood (REML) analyses using the QUERCUS program of Shaw \& Shaw (1992). All variance components were constrained to non-negative values, and the significance of each component (except environmental) was tested by a likelihood-ratio test (Shaw \& Shaw, 1992). The bio model assumes that there is no epistasis and excludes genotype-environment interactions. Data were examined for normality, and the transformation (seed number) ${ }^{3}$ and the angular transformation of fruit-set were selected.

The bio model takes into account that each parent acts in both maternal and paternal roles in the diallel (Waser et al., 1995). It enables estimation of six variance components and minimizes the confounding of genetic and environmental effects. This is advantageous when compared to other analytic models, such as factorial ANOVAS, which can only produce four variance components: maternal, paternal, maternal-paternal interaction, and residual environmental variance (Shaw \& Waser, 1994). The applicability of the bio model to postpollination traits, however, is more limited than for other traits. For postpollination traits, variance components can be affected not only by genes expressed in the progeny generation, but also by genes expressed in the haploid male and female gametophytes and the triploid endosperm. Thus, the component estimates from the bio model reflect the contribution of specific combinations of parents, as well as the genotype of the progeny, and must be interpreted with caution. Moreover, estimates of $V_{\text {environmental }}$ reflect solely the variance of residuals within cells of the diallels. Additional environmental variation may exist, however, but will be confounded 
with the other variance components (see Waser et al., 1995 for discussion). For example, plants receiving more nutrients might be bigger and, consequently, produce fruits with greater seed mass. Such variation would appear in the estimate of $V_{\text {maternal }}$, not $V_{\text {environmental. }}$. Likewise, estimates of $V_{\text {joint }}$ reflect the covariance of individuals in their maternal and paternal functions and, thus, variation caused by environmental effects expressed in both male and female tissues will be reflected in $V_{\text {joint }}$. Similar arguments can be extended to estimates of $V_{\text {paternal }}$ and $V_{\text {combined }}$.

For comparative purposes, and because fruit-set is not really a trait of the progeny generation, we also analysed these data using a factorial ANOVA. This ANOVA included the effects of female plant, male plant and the female-male interaction and used the SAS macro GLIMMIX, which can be programmed to account for the binomial distribution of fruit-set data. The models analysed included female, male, and female-male interaction as fixed effects and used REML to estimate variance components. Date of pollination was initially tested as a covariate, but it was excluded from the final models because it had no significant effect on the response variables. 'Umbel' was included as a random factor to account for variation among umbels for each female by male combination. Finally, an extra-dispersion factor was calculated, which reflects the conditional error of variance associated with flowers within umbels (see Littell et al., 1996).

The relationship between male and female success of individual plants was also examined for the three postpollination traits. Separate estimates of male and female function were obtained for each plant from the least-squares means of a factorial ANOvA that included only male and female main effects (see model A11 of
Schlichting \& Devlin, 1989). The estimates were then used in Pearson correlation analyses. To test whether variance in reproductive success among females equalled that of males, the same individual estimates of male and female function were treated as observations for Levene's median-ratio test for relative variation (Schultz, 1985; Lyons, 1996). In this test, each variate $X_{i}$ in a set of $i=1,2, \ldots, m$ samples was converted to absolute deviations estimates: $\left|X_{i}-\operatorname{Md}\left(X_{i}\right)\right| \operatorname{Md}\left(X_{i}\right)$, where $\operatorname{Md}\left(X_{i}\right)$ was the median value for the group of $m$ samples. These values were then used in $F$-tests (Schultz, 1985).

\section{Results}

A correlation analysis performed for the three postpollination traits showed no significant partial correlations except for seed mass and number in D1 (Table 1). Surprisingly, this partial correlation was positive, which indicates that fruits producing many seeds contained heavier seeds. For D2, however, the partial correlation between seed mass and number was negative, although it was not significantly different from zero. The mean values for the three reproductive traits and associated coefficients of variation are shown in Table 2.

For both seed mass and seed number, the bio model analyses showed that the observed variance was largely attributable to $V_{\text {maternal }}, V_{\text {interaction }}$ and $V_{\text {environmental }}$, but not to $V_{\text {paternal }}, V_{\text {joint }}$ or $V_{\text {combined }}$ (Table 3). For seed mass, $V_{\text {maternal }}$ was the most important source of variance in D2, accounting for $68.4 \%$ of the total, but it accounted for less in D1 (31.3\%). In D1, $V_{\text {interaction }}$ explained $46.5 \%$ of the variance, but in D2 it explained $7.27 \%$ of variance and was only marginally significant. The contribution of $V_{\text {environmental }}$ was more consistent

Table 1 Partial correlations for pairs of postpollination traits in plants of Asclepias incarnata. Traits correlated were individual seed mass and seed number, fruit-set and seed number, and fruit-set and individual seed mass. $F$-statistics for Wilks's lambda test the hypothesis of no overall male $\times$ female or female effect

\begin{tabular}{lccccr}
\hline & & & \multicolumn{2}{c}{ Wilks's lambda } \\
\cline { 5 - 6 } Diallel & $r$ & d.f. & Effect & $F$ & d.f.1, d.f.2 \\
\hline Seed mass and number & & & & \\
$\quad$ D1 & $0.787^{* * *}$ & 98 & Female $\times$ male & $7.22^{* * *}$ & 34,194 \\
D2 & -0.197 & 40 & Female $\times$ male & $2.51^{* * *}$ & 18,78 \\
Fruit-set and seed number & & & & \\
D1 & 0.303 & 22 & Female & $2.26^{*}$ & 10,42 \\
D2 & 0.065 & 13 & Female & $3.80^{* *}$ & 8,24 \\
Fruit-set and seed mass & & & & $3.05^{* *}$ & 10,42 \\
D1 & 0.290 & 22 & Female & $6.74^{* * *}$ & 8,24 \\
D2 & 0.326 & 13 & Female &
\end{tabular}

${ }^{*} P<0.05 ;{ }^{* *} P<0.01 ;{ }^{* * *} P<0.001$; d.f.1, numerator d.f.; d.f.2, denominator d.f.

(C) The Genetical Society of Great Britain, Heredity, 83, 310-318. 
Table 2 Mean values (and associated coefficients of variation) for fruit-set, individual seed mass, and seed number from two diallel crosses among plants of Asclepias incarnata

\begin{tabular}{lccc}
\hline Diallel & Fruit-set & Seed mass $(\mathrm{mg})$ & Seed number \\
\hline D1 & $0.299(0.956)$ & $7.06(0.19)$ & $58.4(0.18)$ \\
D2 & $0.176(1.110)$ & $6.49(0.17)$ & $53.9(0.17)$ \\
\hline
\end{tabular}

$(22.2 \%$ and $17.5 \%$ of total variance in D1 and D2, respectively). For seed number, $V_{\text {interaction was the }}$ largest source of variation in both diallels; it was highly significant and accounted for $31.4 \%$ and $39.1 \%$ of total variance in D1 and D2, respectively. $V_{\text {maternal }}$ explained some of the variation in seed number $(20.5 \%$ and $37.1 \%$ ), but this contribution was not significant in D1 and only marginally so in D2. Again, the contribution of $V_{\text {environmental to total variance was consistent and }}$ important (34.1\% and $23.9 \%)$.

The results from the bio analysis of fruit-set differed substantially from those of the GLIMMIX analysis (cf. Tables 3 and 4). In the bio analysis, the estimate of

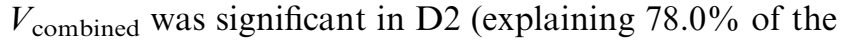
variance) and was marginally significant in D1 (explaining $22.9 \%$ of the variance). The $V_{\text {maternal }}$ estimate was also significant for D2, but not for D1, and accounted for $7.5 \%$ and $0.7 \%$ of the variation, respectively. $V$ environmental explained $26.2 \%$ and $10.1 \%$ of total variation in D1 and D2. With GLIMMIX, however, no significant female-male interaction was found (Table 4).
Moreover, the estimates of female variance were significant in both diallels, and the estimate of paternal variance was significant in D1. The GLIMMIX model also revealed that flowers within umbels acted essentially independently with respect to fruit-set (indicated by the dispersion values of 0.79 and 0.92 for D1 and D2), but the random effect estimates showed that fruit-set varied between umbels.

For both seed traits, performance of individuals as males was not correlated significantly with their performance as females (Table 5). This lack of correlation, however, must be viewed cautiously, because the sample sizes were small. The variance among plants for female function, however, tended to be greater than their variance for male function as determined by Levene's test (Table 5). This increased variance was significant for seed number in D2, for seed mass in D1, and nearly significant for seed mass in D2. For fruit-set, on the other hand, male and female success were positively correlated in both diallels, but the correlation was significant only in D2. The variance in success among plants as females did not differ from their variance as males.

\section{Discussion}

Maternal variation, which reflects differences between plants acting as pollen recipients, accounted for significant portions of the variance in fruit-set, seed number and seed mass in the individuals of Asclepias incarnata that we studied. Results from numerous other studies

Table 3 Relative percentage of six causal components of variance out of total phenotypic variance for three postpollination traits estimated after crossing individuals of Asclepias incarnata in diallels

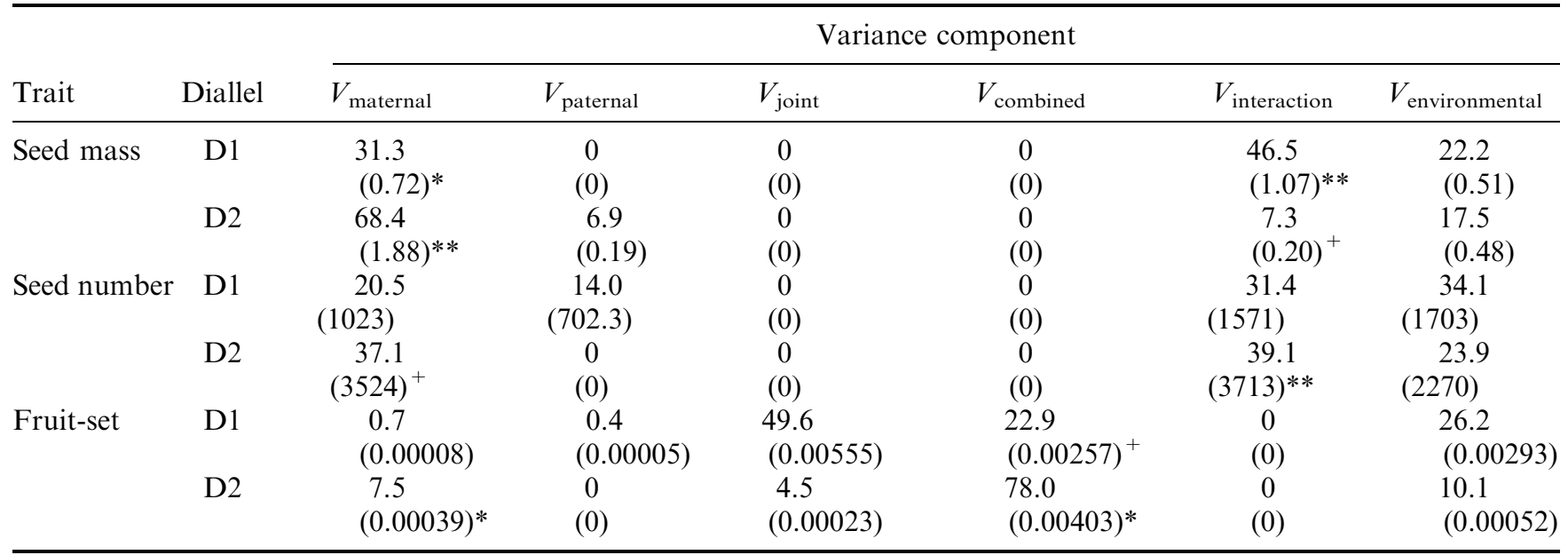

The values of the restricted maximum-likelihood estimates for the variance components are shown in parentheses. Zeroes indicate components that were constrained to zero, after initial negative estimates. Significance of individual components (except $V_{\text {environmental }}$ ) was tested using a likelihood-ratio test.

${ }^{+} P<0.1,{ }^{*} P<0.05,{ }^{* *} P<0.0001$. 
Table 4 Effects of female and male plants, and the interaction between these main effects, on fruit-set from plants of Asclepias incarnata crossed in two diallels. Data from D1 and D2 were analysed separately using a generalized linear mixed-model analysis that included umbel as a random effect

\begin{tabular}{llccc}
\hline Diallel & Source & d.f. & Type III $F$ & $P$ \\
\hline Fixed effects & & & & \\
D1 & Female & 5 & 4.27 & 0.0013 \\
& Male & 5 & 4.91 & 0.0004 \\
& Female $\times$ male & 17 & 1.17 & 0.2986 \\
D2 & Female & 4 & 8.02 & 0.0001 \\
& Male & 4 & 0.32 & 0.8679 \\
& Female $\times$ male & 10 & 0.86 & Mean value \\
\hline Diallel & Deviance (d.f.) & Umbel (d.f.) & Dispersion & 0.299 \\
\hline Model and random effects & & & & 0.79 \\
D1 & $728(868)$ & $1.18(144)$ & 0.92 & \\
D2 & $474(603)$ & $0.28(101)$ & & \\
\hline
\end{tabular}

Table $5 \mathrm{~F}$-tests comparing variation in male vs. female success and a correlation analysis of male and female success for plants of Asclepias incarnata that were crossed in diallels. The $F$-tests were based on Levene's median-ratio deviates

\begin{tabular}{|c|c|c|c|c|c|c|c|c|c|}
\hline \multirow[b]{2}{*}{ Trait } & \multirow[b]{2}{*}{ Diallel } & \multicolumn{5}{|c|}{ Median-ratio test } & \multicolumn{3}{|c|}{ Correlation analysis } \\
\hline & & d.f. & Male lsmean & Female lsmean & $F$ & $P$ & $N$ & $r$ & $P$ \\
\hline \multirow[t]{2}{*}{ Seed number } & D1 & 5,5 & 0.0447 & 0.0647 & 2.04 & 0.227 & 6 & -0.00025 & 1.000 \\
\hline & D2 & 4,4 & 0.0154 & 0.1074 & 77.99 & 0.0005 & 5 & -0.629 & 0.255 \\
\hline \multirow[t]{2}{*}{ Seed mass } & D1 & 5,5 & 0.0297 & 0.0956 & 10.47 & 0.0111 & 6 & -0.485 & 0.330 \\
\hline & D2 & 4,4 & 0.0650 & 0.1541 & 5.65 & 0.0610 & 5 & -0.178 & 0.775 \\
\hline \multirow[t]{2}{*}{ Fruit-set } & D1 & 5,5 & 0.2965 & 0.2905 & 0.65 & 0.325 & 6 & 0.674 & 0.142 \\
\hline & D2 & 4,4 & 0.2064 & 0.3665 & 3.47 & 0.1277 & 5 & 0.986 & 0.002 \\
\hline
\end{tabular}

suggest that environmental factors that influence plant size or resource status are the major causes of this maternal variation (Bookman, 1984; Roach \& Wulff, 1987). Environmental factors were probably important in our diallels as well, even though the crosses were conducted in the glasshouse to minimize differences between plants. Some of the maternal variation, however, may have been heritable (Platenkamp \& Shaw, 1993). For example, nuclear genes might have affected ovule number and size and could have contributed to $V_{\text {maternal }}$ but not to $V_{\text {joint }}$. Another possible cause of maternal variation is the expression of extranuclear genes that are maternally inherited by developing seeds (Roach \& Wulff, 1987; Falconer, 1989). Some of the variation that contributed to $V_{\text {maternal }}$, however, may not reflect true differences between plants acting as pollen recipients. Instead, $V_{\text {maternal }}$ could be confounded with genetic variation from haploid gametophytic or triploid endosperm tissues alone or in combination with diploid maternal tissues (Shaw \& Waser, 1994). Unfortunately, multigenerational crossing designs are required to evaluate the importance of these nonstandard genetic entities (Cockerham \& Weir, 1977; Shaw \& Waser, 1994).

Most studies, including this one, report that maternal variance contributes much more than paternal variance to total phenotypic variance (Antonovics \& Schmitt, 1986; Mazer, 1987a,b; Schlichting \& Devlin, 1989; Schwaegerle \& Levin, 1990; Morse \& Schmitt, 1991; Montalvo \& Shaw, 1994; Waser et al., 1995; Lyons, 1996). This gender-specific difference is reflected in the results from the median ratio tests, which show that variation in female function exceeded variation in male function for seed number and mass. Moreover, in the bio analyses, none of the estimates of paternal variation was statistically different from zero. This indicates that differences between pollen donors were minimal. Assuming that differences between males are not much greater in natural populations than in this glasshouse study, these results suggest that selection is unlikely to act directly on postpollination processes that determine 
pollen donation in A. incarnata. It is notable that studies applying the bio model rarely ascribe significant portions of variance to male function (Antonovics \& Schmitt, 1986; Schwaegerle \& Levin, 1990; Biere, 1991; Morse \& Schmitt, 1991; Montalvo \& Shaw, 1994; Waser et al., 1995; Lyons, 1996), but those analysing models that include only the effects of male and female and the malefemale interaction often ascribe significant portions to it, for at least some traits (Marshall \& Ellstrand, 1986; Mazer, 1987b; Schlichting \& Devlin, 1989; Lyons, 1996).

No evidence of significant variation attributable to the joint contribution of parents through male or female function $\left(V_{\text {joint }}\right)$ was detected for any trait in the individuals studied. Several other studies have also failed to find significant contributions of $V_{\text {joint }}$ to phenotypic variance for seed mass and number (Antonovics \& Schmitt, 1986; Mazer, 1987b; Schwaegerle \& Levin, 1990; Waser et al., 1995). This is not surprising, given the limitations of estimates of $V_{\text {joint }}$ for postpollination traits. Postpollination traits may reflect properties of both parental and progeny generations, but the joint variance estimate includes only that portion of the variance that does not reflect parental contributions. Thus, single-generation estimates for postpollination traits cannot reveal whether additive genetic variation exists, on which selection may act.

The estimate of combined variation was significant for fruit-set in one diallel and nearly significant in the second diallel. It reflects differences in fruit production from specific combinations of plants, regardless of which plant serves as pollen donor or as pollen recipient. This interaction between plants for fruit-set could be related to self-incompatibility, inbreeding depression, and/or 'embryo control'. The populations of $A$. incarnata that we studied cannot easily be categorized as selfcompatible or self-incompatible, as they have natural outcrossing rates near $100 \%$ but contain a mixture of self-fertile and self-sterile plants (Lipow, 1998; Ivey et al., 1999). The plants could possess the unusual postzygotic self-incompatibility system, controlled by one gene, found in A. exaltata (Lipow, 1998). Asclepias exaltata also displays plant-to-plant variation in the success of self- vs. cross-pollinations, and this variation has been shown to result from the effects of pseudo-selffertility alleles at genes other than the self-incompatibility locus (Lipow et al., 1999). If self-incompatibility and pseudo-self-fertility occur in A. incarnata as well, then the observed combined variation probably results from self-incompatibility alleles or pseudo-self-fertility alleles shared between some plants. Alternatively, the variation in self- and cross-fertility in $A$. incarnata could be caused by the effects of inbreeding depression on embryo development. If this is the case, then the observed combined variation probably reflects differen- ces in relatedness between pairs of plants that result in differences in inbreeding depression. Finally, "embryo control' over fruit production via hormonal signals or patterns of resource acquisition could contribute to the observed combined variation. Such embryo control cannot involve 'female choice' (i.e. maternal discrimination among progeny or paternal genotypes by pollen recipients), because the differences between crosses did not depend on which plant served as pollen donor or as pollen recipient (Morse \& Schmitt, 1991). Instead, embryo control reflects differences based only on the genotypes of the embryos within the fruits.

The combined variation that we detected sheds light on the unusually low fruit-set of milkweeds, which typically ranges from $0.33 \%$ to $7.0 \%$ after natural pollination (Wilbur, 1976). Two hypotheses have been proposed to explain this low fruit-set (reviewed by Wyatt \& Broyles, 1994): (i) resources to mature fruits are limiting; and (ii) flowers receive insufficient numbers of cross-pollinations. Combined variation for fruit-set, however, indicates that fruit maturation following crosspollination is determined not only by the resource status of the plants, but also by the 'combining ability' of the cross. This blurs the distinction between the pollen and resource limitation hypotheses. With combined variation, two plants with identical resource status that receive the same number of cross-pollinations with pollen from the same donors could mature very different numbers of fruits. Combined variation also helps to explain why fruit-set after cross-pollinations and under ideal conditions in the glasshouse rarely exceeds $50 \%$ (Wyatt \& Broyles, 1994; Ivey et al., 1999).

We know of only two other studies in which the bio model was applied to fruit-set. One of these involved 10 individuals of another milkweed, A. syriaca, and found, as we did, significant maternal (termed 'maternal reciprocal general') and combined ('nuclear specific') effects (Morse \& Schmitt, 1991). The similarity of the results supports our belief that the genetic interactions that manifest in combined variation are related to the low fruit-set characteristic of all milkweeds. The only other analysis of fruit-set (Lyons, 1996) involved two populations of Leavenworthia crassa, in which only significant maternal effects were detected. Clearly, further studies of variation in fruit-set are warranted.

Despite the significant estimates for combined variation from the bio analysis, the estimates of female-male interactions from the GLIMMIX analyses of fruit-set were not significant in either diallel. The bio model approach assumes that the causal variance components are linked in a specified way to the observational variances arising from differences between and within full-sib and half-sib families, with some components being a complex function of more than one level in the family structure. 
These links can lead to unpredictable effects on the variance estimates when there are sources of variation not accounted for by the model. The GLIMMIX approach, however, does not assume a particular genetic model. Thus, a possible explanation for the discrepancy between the two approaches is that variation embodied by $V_{\text {combined }}$ and $V_{\text {interaction }}$ are entirely confounded in the GLIMMIX analysis and may partially cancel each other. Additionally, effects caused by haploid pollen would appear as paternal effects in the GLIMMIX analysis, but they could be partitioned in $V_{\text {combined }}$ in the bio model.

Asymmetrical interactions ( $\left.V_{\text {interaction }}\right)$, in addition to maternal effects, accounted for significant variation in seed number and mass. These interactions reflect differences in seed number and mass from specific combinations of plants that depend on which plant serves as pollen donor vs. pollen recipient. They could be caused by the differential provisioning of resources by maternal plants to seeds (or fruits containing seeds) in response to the genotypes of the zygotes they contain. Alternatively, maternal plants could respond to the specific genotypes of the pollen that fertilized specific ovules or ovaries. Both of these interactions involve the maternal genotype and could be viewed as forms of 'sexual selection', because they amount to either 'female choice' or competition among embryos sired by different males. There are, however, other possible causes of $V_{\text {interaction }}$. Interactions occurring within zygotes between nuclear genes of one parent and cytoplasmic genes of the other, or between cytoplasmic genes of both parents, could affect the survival and vigour of the zygotes and, hence, contribute to $V_{\text {interaction }}$.

A few other studies have also identified significant estimates of $V_{\text {interaction }}$ for seed mass (Schwaegerle \& Levin, 1990; Biere, 1991; Montalvo \& Shaw, 1994), but never at a magnitude as great as we found $(7-47 \%$ of total phenotypic variation). For example, of the variation in seed mass, $V_{\text {interaction }}$ explained only $2 \%$ in Phlox drummondii (Schwaegerle \& Levin, 1990), 3-4\% in Aquilegia caerulea (Montalvo \& Shaw, 1994), and 6\% in Lychnis flos-cuculi (Biere, 1991). Ours is also the first report of significant $V_{\text {interaction }}$ for seed number. The highly significant male-female interaction reported for seed number by Schlichting \& Devlin (1989), however, could indicate similar asymmetrical effects, but this cannot be determined with certainty, because their experimental design confounded $V_{\text {interaction }}$ with $V_{\text {combined }}$ and $V_{\text {joint }}$.

The unusually high estimate of $V_{\text {interaction }}$ for seed mass and number in this study may be related to the enclosure of milkweed pollen grains in pollinia. We suspect that environmental variation between individual pollen grains in pollinia is much lower than that between loose pollen grains of other plants. Moreover, pollinia reduce variation in the number of pollen grains used per pollination event, relative to plants with loose pollen grains. These factors probably decreased environmental variation in seed number and, thereby, increased our ability to detect genetic sources of variation. Moreover, because of the enclosure of pollen in pollinia, a single pollen donor typically fertilizes all of the ovules within a milkweed fruit (Wyatt \& Broyles, 1994). Thus, any differential provisioning of resources by maternal plants to developing seeds based on the genotypes of the zygotes the seeds contain could be amplified, because all of the seeds within a fruit are related as full-siblings. For example, fruits that contain many embryos with favourable genotypes could receive a disproportionate share of maternal resources and, consequently, all seeds within the fruit might be heavier than average. Further investigation into the precise genetic mechanisms responsible for $V_{\text {interaction }}$ would be of interest.

The significant estimates of interaction effects for seed mass and number $\left(V_{\text {interaction }}\right)$ and fruit-set ( $\left.V_{\text {combined }}\right)$ indicate that male and female function are linked, regardless of the underlying genetic mechanisms. Because of these interactions, selection cannot independently influence male or female function, despite the nonsignificant but slightly negative correlation between these functions for seed mass and number. Indeed, the failure to detect a significant correlation between the functions may result from small sample sizes. Furthermore, variation in female success exceeded variation in male success; thus, if selection occurs on these traits, it may operate more strongly on female function than on male function. These results contrast with the conventional idea that there should be a trade-off between male and female function and suggest instead that male and female functions will evolve together.

\section{Acknowledgements}

We thank S. Andersson, C. Ivey, J. Hamrick and S. Mazer for comments on earlier versions of this manuscript; R. G. Shaw for assistance with QUERCUs; G. Derda for computer support; and A. Tull, M. Zimmerman and M. Smith for excellent glasshouse care. This paper represents a portion of a Ph.D. Dissertation submitted to the University of Georgia. Funding was provided by National Science Foundation Grant no. DEB-9623925 and the University of Georgia Botany Department Small Grants Program.

\section{References}

ANTONOvics, J. AND SCHMitT, J. 1986. Paternal and maternal effects on propagule size in Anthoxanthum odoratum. Oecologia, 69, 277-282. 
BIERE, A. 1991. Parental effects in Lychnis flos-cuculi. I. Seed size, germination and seedling performance in a controlled environment. J. Evol. Biol., 3, 447-465.

BOOKMAN, S. S. 1984. Evidence for selective fruit production in Asclepias. Evolution, 38, 72-86.

COCKERHAM, C. C. AND WEIR, B. S. 1977. Quadratic analyses of reciprocal crosses. Biometrics, 33, 187-203.

FALCONER, D. S. 1989. Introduction to Quantitative Genetics, 3rd edn. John Wiley, New York.

IVEY, C. I., LIPOW, S. R. AND WYATT, R. 1999. Mating systems and interfertility of swamp milkweed (Asclepias incarnata ssp. incarnata and ssp. pulchra). Heredity, 82, 25-35.

LIPOW, S. R. 1998. Post-zygotic Self-incompatibility and Self-fertility in the Apocynaceae and Asclepiadaceae. Ph.D. Dissertation, University of Georgia.

LiPOW, S. R., BROYLES, S. B. AND WYATT R. 1999. Population differences in self-fertility in the "self-incompatible" milkweed Asclepias exaltata (Asclepiadaceae). Am. J. Bot. (in press).

LITTELl, R. C., MILliKeN, G. A., STROUP, W. W. AND WOLFINGER, R. D. 1996. SAS System for Mixed Models. SAS Institute Inc., Cary, NC.

LYONS, E. E. 1996. Breeding system evolution in Leavenworthia. II. Genetic and nongenetic parental effects on reproductive success in selfing and more outcrossing populations of Leavenworthia crassa. Am. Nat., 147, 65-85.

LYONS, E. E., WASER, N. M., PRICE, M. V., ANTONOVICS, J. AND MOTTEN, A. F. 1989. Sources of variation in plant reproductive success and implications for concepts of sexual selection. Am. Nat., 134, 409-433.

MARSHALL, D. L. AND ELLSTRAND, N. C. 1986. Sexual selection in Raphanus sativus: experimental data on nonrandom fertilization, maternal choice, and consequences of multiple paternity. Am. Nat., 127, 446-461.

MAZER, S. J. 1987a. The quantitative genetics of life history and fitness components in Raphanus raphanistrum L. (Brassicaceae): ecological and evolutionary consequences of seedweight variation. Am. Nat., 130, 891-914.

MAZER, S. J. 1987b. Parental effects on seed development and seed yield in Raphanus raphanistrum: implications for natural and sexual selection. Evolution, 41, 355371.
MONTALVO, A. M. AND SHAw, R. G. 1994. Quantitative genetics of sequential life-history and juvenile traits in the partially selfing perennial Aquilegia caerulea. Evolution, 48, 828-841. MORGAN, M. T. 1994. Models of sexual selection in hermaphrodites, especially plants. Am. Nat., 144, S100-S125.

MORSE, D. H. AND SCHMITT, J. 1991. Maternal and paternal effects on follicle production in the milkweed Asclepias syriaca (Asclepiadaceae). Am. J. Bot., 78, 1304-1309.

PLATENKAMP, G. A. J. AND SHAW, R. G. 1993. Environmental and genetic maternal effects on seed characters in Nemophila menziesii. Evolution, 47, 540-555.

ROACH, D. A. AND WULFF, R. D. 1987. Maternal effects in plants. Ann. Rev. Ecol. Syst., 18, 209-235.

SAS INSTITUTE INC. 1987. SAS/STAT Guide for Personal Computers, Version 6 Edn. SAS Institute Inc., Cary, NC.

SCHLichting, C. C. AND DEVlin, B. 1989. Male and female reproductive success in the hermaphroditic plant Phlox drummondii. Am. Nat., 133, 122-227.

SCHUltz, в. в. 1985. Levene's test for relative variation. Syst. Zool., 34, 449-456.

SCHWAEGERLE, K. E. AND LEVIN, D. A. 1990. Quantitative genetics of seed size variation in Phlox. Evol. Ecol., 4, 143-148.

SHAw, R. G. AND SHAw, F. H. 1992. Quercus: Programs for the analysis of quantitative genetic data by maximum likelihood. Published electronically on the Internet, available via anonymous ftp from ftp.bio.indiana.edu; directory path biology/quantgen/quercus.

SHAW, R. G. AND WASER, N. M. 1994. Quantitative genetic interpretations of postpollination reproductive traits in plants. Am. Nat., 143, 617-635.

WASER, N. M., SHAW, R. G. AND PRICE, M. V. 1995. Seed set and seed mass in Ipomopsis aggregata: variance partitioning and inferences about postpollination selection. Evolution, 49, 80-88.

WILBUR, H. M. 1976. Life history evolution in seven milkweeds of the genus Asclepias. J. Ecol., 64, 223-240.

WILSON, P., THOMSON, J. D., STANTON, M. L. AND RIGNEY, L. P. 1994. Beyond floral Batemania: gender biases in selection for pollination. Am. Nat., 143, 283-296.

WYATT, R. AND BROYLES, S. B. 1994. Ecology and evolution of reproduction in milkweeds. Ann. Rev. Ecol. Syst., 25, 423441. 